\title{
FUNGOS MICORRÍZICOS ARBUSCULARES EM ÁREAS DE PLANTIO DE LEUCENA E SÁBIA NO ESTADO DE PERNAMBUCO ${ }^{1}$
}

Luciana Xavier da Silva², Márcia do Vale Barreto Figueiredo², Gladstone Alves da Silva ${ }^{3}$, Bruno Tomio Goto $^{4}$, José de Paula Oliveira ${ }^{2}$ e Hélio Almeida Burity ${ }^{2}$

\begin{abstract}
RESUMO - A ocorrência de fungos micorrízicos arbusculares (FMAs) foi investigada em áreas cultivadas com leucena (Leucaena leucocephala L.) e sabiá (Mimosa caesalpiniaefolia Benth), no Estado de Pernambuco. Amostras de solo foram coletadas em cinco estações experimentais da Empresa Pernambucana de Pesquisa Agropecuária (IPA), nas cidades de Arcoverde, com plantio de leucena (AL) e sabiá (AS); Caruaru, com plantio de leucena (CL); e Itambé (IS), São Bento do Una (SBS) e Serra Talhada (STS), com plantio de sabiá. O número de esporos variou de 69 (STS) a 437/50 g de solo (CL) e a colonização micorrízica, de 11 (CL) a 64\% (IS). Nas áreas com plantio de leucena, a colonização foi inferior a 15\%, enquanto naquelas com cultivo de sábia houve variação de 36 a 64\%. Foram observadas 24 espécies de FMAs, pertencentes aos gêneros: Acaulospora (6), Archaeospora (1), Entrophospora (1), Gigaspora (3), Glomus (6), Paraglomus (1) e Scutellospora (6). As áreas CL e STS foram as que apresentaram maior número de espécies (14). Acaulospora scrobiculata, Archaeospora leptoticha e Glomus etunicatum foram encontradas em todas as áreas.
\end{abstract}

Palavras-chave: Glomeromycota, Leucaena leucocephala e taxonomia.

\section{ARBUSCULAR MYCORRHIZAL FUNGI IN PLANTATIONS OF LEUCAENA AND MIMOSA IN PERNAMBUCO STATE}

\begin{abstract}
The occurrence of arbuscular mycorrhizal fungi $(A M F)$ in plantations of Leucaena leucocephala L. (leucaena) and Mimosa caesalpiniaefolia Benth (sabia) in the State of Pernambuco was investigated. Soil samples were collected in five research centers of the Agricultural Research Institute of Pernambuco (IPA), in the cities of Arcoverde - plantation of leucaena (AL) and sabiá (AS); Caruaru (CL) - leucaena plantation; Itambé (IS), São Bento do Una (SBS) and Serra Talhada (STS) - plantation of sabiá. Mycorrhizal spores were extracted from soil, with the highest number (437/50g of soil) being found in the CL region and the lowest (69/50g of soil) in the STS region. The mycorrhizal colonization ranged from $11 \%$ (CL) to $64 \%$ (IS). The colonization was inferior to $15 \%$ in areas of leucaena plantation, however, in areas of sabiá, it ranged from $36 \%$ to $64 \%$. Twenty-four AMF species were identified in the genus: Acaulospora (6), Archaeospora (1), Entrophospora (1), Gigaspora (3), Glomus (6), Paraglomus (1) and Scutellospora (6). The highest species number was recorded in the CL and STS regions (14). Acaulospora scrobiculata, Archaeospora leptoticha and Glomus etunicatum were found in all regions.
\end{abstract}

Keywords: Glomeromycota, Leucaena leucocephala and taxonomy.

\footnotetext{
${ }^{1}$ Recebido em 20.10.2006 e aceito para publicação em 08.01.2007.

${ }^{2}$ Empresa Pernambucana de Pesquisa Agropecuária. Av. General San Martin, 1371, Bonji, 50761-000 Recife-PE. E-mail: <luciana_xs@yahoo.com.br>; <mbarreto@elogica.com.br>; <jpaula@ipa.br>; <halmeida@ipa.br>.

${ }^{3}$ Centro Federal de Educação Tecnológica do Piauí - Uned Floriano. Rua Francisco Urquiza Machado, 462, Meladão, 64800-000 Floriano-PI. E-mail: <gladstonesilva@yahoo.com>.

${ }^{4}$ Universidade Federal de Pernambuco. Av. Nelson Chaves, S/N, Várzea, 50670-420 Recife-PE. Email: <brunogoto@ hotmail.com>
} 


\section{INTRODUÇÃO}

A simbiose micorrízica contribui para a sobrevivência e crescimento das espécies, principalmente em ambientes estressantes (SIQUEIRA e SAGGIN-JUNIOR, 1995), onde os fungos micorrízicos arbusculares (FMA) exercem grande influência na estruturação das comunidades vegetais (SIQUEIRA et al., 1994), com o aumento na absorção de nutrientes de baixa mobilidade no solo (MARSCHNER eDELL, 1994) e na tolerância das plantas a doenças radiculares (MUNYANZIZA et al., 1997); melhoram a estrutura do solo, favorecendo a sua retenção de umidade, a agregação e estabilidade(AUGÉ et al., 2001); e influenciam a diversidade e a produtividade vegetal (MILLER e KLING, 2000).

Os FMAs, além de aumentarem a capacidade das plantas em absorver água e nutrientes, são capazes de tolerar estresses diversos, como aqueles causados por metais tóxicos do solo (SIQUEIRA et al., 1999). Diante da necessidade de revegetar ou da possibilidade de usar plantas no processo de descontaminação do solo (BAKER et al., 1994), as interações de micorrizas e leguminosas tornam-se de grande importância na restauração de ecossistemas (DIAZ et al., 1996).

Por causa desses benefícios, os FMAs apresentam grande potencial biotecnológico, possibilitando maior vantagem competitiva das plantas micorrizadas e facilitando o estabelecimento e sucessão da vegetação, o que contribui para a reabilitação de áreas degradadas. Além disso, o manejo apropriado pode reduzir a utilização de fertilizantes e pesticidas químicos (FOCCHI et al., 2004).

O uso de microrganismos com a finalidade de melhorar a disponibilidade de nutrientes às plantas é prática de grande importância e muito necessária para a agricultura. Entre os sistemas biológicos envolvendo planta e microrganismos, têm-se as simbioses leguminosas-rizóbios, de maior expressão econômica, e leguminosas-FMAs, que, a partir de estudos de Gerdermann (1975), também foi considerada importante para o processo de nodulação.

Muitas leguminosas, incluindo leucena e sabiá, são capazes de formar nódulos com bactérias fixadoras de nitrogênio e têm potencial de uso em sistemas agroflorestais, para reabilitação de áreas degradadas e auxiliar a manutenção da sustentabilidade dos solos (FRANCO e FARIA, 1997).
O sabiá (Mimosa caesalpiniifolia Benth.) é uma das mais importantes leguminosas tropicais arbóreas, pela sua comprovada resistência à seca e rápido crescimento (ALMEIDA et al., 1987) e por ser considerada indispensável em qualquer programa de reflorestamento na Região Nordeste, principalmente no semi-árido.

O uso de leucena como cobertura de solo e adubação verde tem-se difundido devido à capacidade que essa planta apresenta de produzir grande quantidade de matéria seca e revegetar solos degradados. Essas características estão principalmente relacionadas à formação de simbiose com bactérias fixadoras de nitrogênio atmosférico e com fungos micorrízicos. Esses microrganismos, quando em simbiose, aumentam a eficiência da planta em usar água e nutrientes, especialmente N e P (BATINI et al., 1994).

Os efeitos dos FMAs sobre o crescimento e a nodulação de leguminosas já são bem conhecidos (GUZMAN-PLAZOLAe FERRERA-CERRATO, 1990). Em leguminosas arbóreas, a presença de micorrizas pode contribuir para expandir a área de captação do $\mathrm{P}, \mathrm{Mo}, \mathrm{Zn}$ e outros nutrientes que chegam até as raízes pelo processo de difusão, permitindo o crescimento em solos extremamente pobres e deficientes em nitrogênio (JESUS et al., 2005).

É considerável o número de trabalho sobre levantamentos de FMAs em diferentes ecossistemas brasileiros, sejam nativos (SOUZA et al., 2003; SILVA et al., 2005) ou uma área impactada (KLAUBERG-FILHO et al., 2002; CAPRONI et al., 2005). Isso porque o conhecimento sobre espécies de FMAs ocorrentes em leguminosas no Nordeste do Brasil ainda é escasso. Assim, este trabalho teve por objetivo registrar a ocorrência de fungos micorrízicos arbusculares em áreas cultivadas com leucena (Leucaena leucocephala L.) e sabiá (Mimosa caesalpiniaefolia Benth) no Estado de Pernambuco.

\section{MATERIAL E MÉTODOS}

As coletas foram realizadas em plantios de leucena (Leucaena leucocephala L.) e sabiá (Mimosa caesalpiniaefolia Benth) no Estado de Pernambuco, em cinco estações experimentais da Empresa Pernambucana de Pesquisa Agropecuária (IPA), localizadas nas cidades de Arcoverde (latitude de $8^{\circ} 25^{\prime}$ $08^{\prime}$ ' $\mathrm{S}$, longitude de $37^{\circ} 03^{\prime} 00^{\prime \prime}$ WGR e altitude de 650 m) com plantio de leucena (AL) e sabiá (AS); Caruaru 
(latitude de $08^{\circ} 14^{\prime} 18^{\prime \prime} \mathrm{S}$, longitude de $35^{\circ} 55^{\prime} 20^{\prime \prime}$ WGR e altitude de $537 \mathrm{~m}$ ), com plantio de leucena (CL); Itambé (latitude de $7^{\circ} 25^{\prime} 0^{\prime \prime} \mathrm{S}$, longitude de $35^{\circ} 6^{\prime} 0^{\prime \prime} \mathrm{WGR}$ e altitude de $190 \mathrm{~m}$ ), com plantio de sabiá (IS); São Bento do Uma (latitude de $08^{\circ} 31^{\prime} 16^{\prime \prime}$, longitude de 36 33' 00", WGR e altitude de $650 \mathrm{~m}$ ), com plantio de sabiá (SBS); e Serra Talhada (latitude de $7^{\circ} 59^{\prime} 15^{\prime}$ 'S, longitude de $38^{\circ} 18^{\prime}$ 0" WGR e altitude de $431 \mathrm{~m}$ ), com plantio de sabiá (STS) (Figura 1).

Em cada uma das áreas foi selecionado um quadrante de $1000 \mathrm{~m}^{2}$, sendo coletadas nove amostras de solo e de raízes provenientes de plantas próximas aos pontos de coleta.

A análise do solo foi realizada pelo Laboratório de Fertilidade do Solo da Empresa Pernambucana de Pesquisa Agropecuária (IPA), considerando-se $\mathrm{pH}$, CTC e os seguintes elementos: $\mathrm{P}, \mathrm{Ca}, \mathrm{Mg}, \mathrm{Na}, \mathrm{K}, \mathrm{S}$ e Al (Tabela 1).

As amostras de raízes coletadas foram diafanizadas com $\mathrm{KOH}(10 \%)$ e coradas com azul de Trypan $(0,05 \%)$ (PHILLIPS e HAYMAN, 1970). Algumas raízes muito pigmentadas foram diafanizadas em $\mathrm{H}_{2} \mathrm{O}_{2}$ aquecido a
$90{ }^{\circ} \mathrm{C}$ durante 10 min. O porcentual de colonização foi calculado pelo método da contagem em lâmina (GIOVANETTI e MOSSE, 1980).

Os esporos foram coletados do solo pelo método do peneiramento úmido (GERDERMAN e NICOLSON, 1963), seguido da centrifugação em sacarose $40 \%$ (JENKINS, 1964) e contados diretamente em placas canaletadas, com o auxílio de estereomicroscópio.

Para identificação das espécies foi utilizado o Manual de Identificação de FMA de Schenck e Pérez (1990), a homepage da International Culture Collection of Arbuscular Mycorrhizal Fungi (INVAM), e publicações mais recentes contendo a descrição de novas espécies de FMAs.

A análise de similaridade entre a comunidade de FMA encontrados nos diferentes locais de coleta foi calculada a partir do coeficiente de similaridade de Sørensen (1978).

Os dados de colonização foram transformados em arco-seno e os de número de esporos, em $\log (\mathrm{x}+1)$, como sugerido por Sieverding (1991), e submetidos à análise de variância pelo teste de Duncan $(\mathrm{p} \leq 0,01)$.
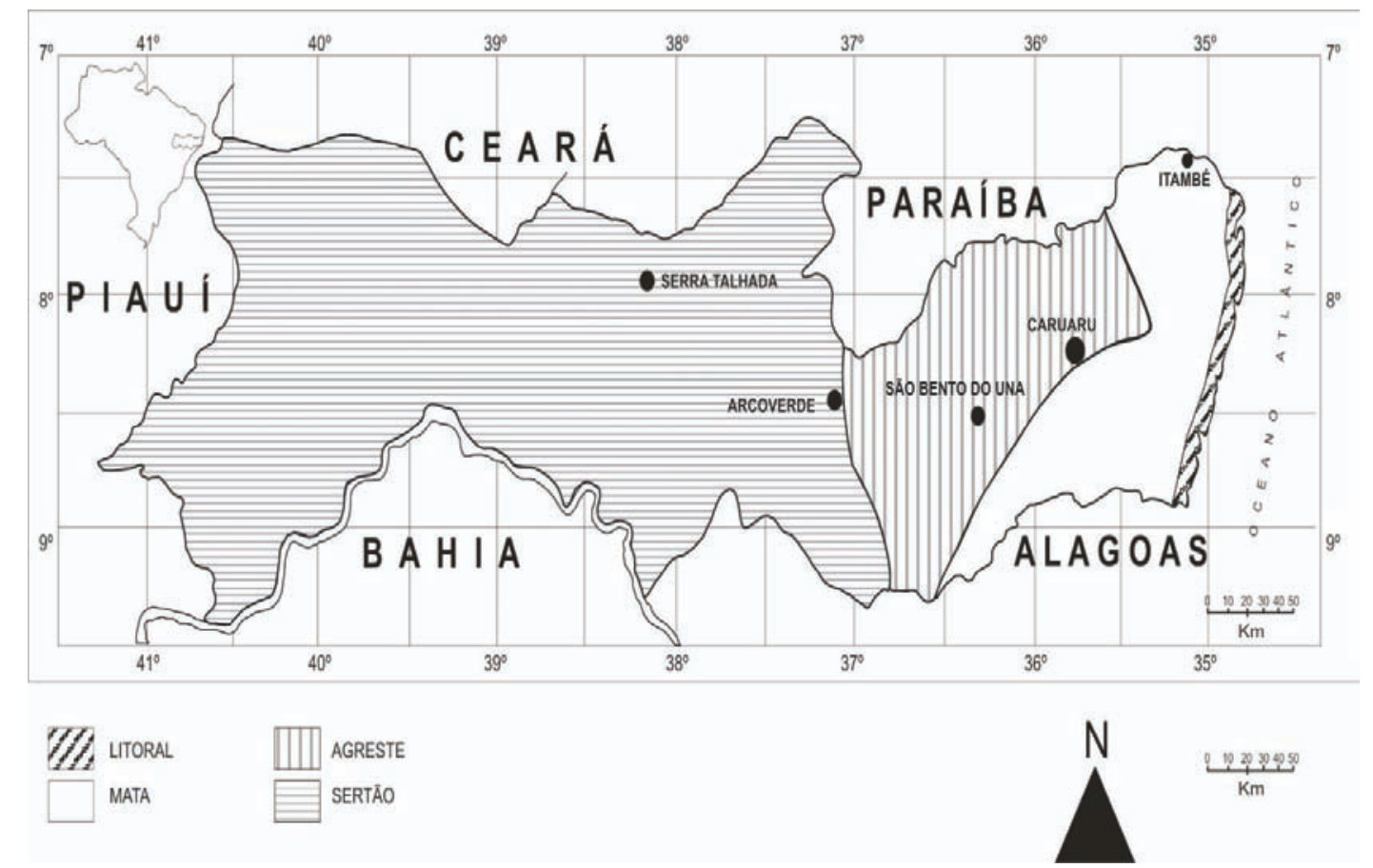

Figura 1 - Locais de coleta no Estado de Pernambuco.

Figure 1 -Sites of collection in the State of Pernambuco 
Tabela 1 - Análise química do solo em áreas com plantio de leucena e sabiá no Estado de Pernambuco Table 1 - Soil chemical analysis in areas with leucena and sabia plantations in the State of Pernambuco

\begin{tabular}{|c|c|c|c|c|c|c|c|c|c|}
\hline \multirow[t]{2}{*}{ Áreas ${ }^{1}$} & $\mathrm{P}$ & $\mathrm{Ca}$ & $\mathrm{Mg}$ & $\mathrm{Na}$ & $\mathrm{K}$ & $\mathrm{Al}$ & $\mathrm{S}$ & CTC & \multirow{2}{*}{$\frac{\mathrm{pH}}{\mathrm{H}_{2} \mathrm{O} 1: 2,5}$} \\
\hline & $\mathrm{mg} / \mathrm{dm}^{3}$ & \multicolumn{7}{|c|}{$\mathrm{cmol}_{\mathrm{c}} \mathrm{dm}^{-3}$} & \\
\hline$\overline{\mathrm{AS}}$ & 36 & 2,20 & 1,15 & 0,12 & 0,33 & 0,35 & 3,8 & 9,4 & 4,5 \\
\hline $\mathrm{AL}$ & 36 & 3,85 & 1,45 & 0,07 & 0,53 & 0,05 & 5,9 & 8,8 & 5,5 \\
\hline CL & 6 & 1,55 & 1,50 & 0,10 & 0,32 & 0,05 & 3,5 & 6,6 & 5,5 \\
\hline IS & 4 & 3,10 & 1,70 & 0,05 & 0,21 & 0,05 & 5,1 & 10,8 & 4,8 \\
\hline SBS & 8 & 1,25 & 0,50 & 0,03 & 0,19 & 0,30 & 2,0 & 4,9 & 4,9 \\
\hline STS & 37 & 2,50 & 1,40 & 0,04 & 0,54 & 0,00 & 4,5 & 6,3 & 6,4 \\
\hline
\end{tabular}

${ }^{1}$ AS-Arcoverde/plantio de sabiá; AL-Arcoverde/plantio de leucena; CL-Caruaru/plantio de leucena;

IS-Itambé/plantio de sabiá; SBS - São Bento do Una/plantio de sabiá; e STS - Serra Talhada/plantio de sabiá.

*Em cada coluna as médias seguidas pela mesma letra não diferem estatisticamente entre si, pelo teste de Duncan (p<0,01)

${ }^{1} A S$ - Arcoverde/sabia plantation; AL - Arcoverde/leucena plantation; CL - Caruaru/leucena plantation;

IS-Itambél sabia plantation; SBS-São Bento do una/ sabia plantation; and STS-Serra Talhada/sabia plantation.

*In each column, means followed by the same letter are not statically different by the Duncan test at P<0.01.

\section{RESULTADOS E DISCUSSÃO}

O número de esporos variou de 69 (área STS) a 437 (área CL) por $50 \mathrm{~g}$ de solo (Tabela 2). Nos cultivos de leucena, esse número foi sempre superior a 200/ $50 \mathrm{~g}$ de solo, enquanto, em geral, as áreas com plantio de sabiá apresentaram número inferior a $200 / 50 \mathrm{~g}$ de solo, com exceção da área IS (212/50 g de solo). As áreas com plantio de leucena exibiram diferenças entre si, quando comparadas pelo teste de Duncan $(\mathrm{p}<0,01)$. As áreas CL e STS foram significativamente diferentes das demais, enquanto as AS, AL, IS e SBS se mostraram semelhantes entre si.

Melo et al. (1997), trabalhando em áreas de cultivo de bananeiras em Pernambuco, relataram uma variação de 44 a 271 esporos por $100 \mathrm{~g}$ de solo. Maia e Trufem (1990) observaram, em plantas de interesse econômico, variação de 26 a 437 esporos por $100 \mathrm{~g}$ de solo. Silva et al. (2001) verificaram, em áreas de caatinga nativa e degradadas por mineração, um nível de esporos sempre inferior a 160/100 g de solo. Já, em áreas poluídas com metais pesados, Klauberg-Filho et al. (2002) encontraram número de esporos variando de 8 a 335 esporos por $50 \mathrm{~g}$ de solo, apresentando maiores valores em locais de menor contaminação. Souza et al. (2003) trabalhando em área de caatinga, observaram variação de 34 a 860 esporos por $100 \mathrm{~g}$ de solo. Esses dados, comparados com os resultados do número de esporos deste trabalho, indicam que em áreas cultivadas geralmente é observado maior número de esporos em comparação com as áreas degradadas.
Tabela 2 - Porcentual de colonização radicular e número médio de esporos de fungos micorrízicos arbusculares (FMAs) por $50 \mathrm{~g}$ de solo em áreas com plantio de leucena e sabiá, no Estado de Pernambuco

Table 2-Percentage of root colonization and mean number of spores of FMAs per $50 \mathrm{~g}$ of soil in areas with leucena and sabia plantations, in the State of Pernambuco

\begin{tabular}{lcc}
\hline Áreas $^{1}$ & $\mathrm{~N}^{\circ}$ de esporos/50g de solo* & Colonização (\%) \\
\hline AS & $184 \mathrm{a}$ & $54 \mathrm{ab}$ \\
AL & $210 \mathrm{a}$ & $14 \mathrm{c}$ \\
CL & $437 \mathrm{~b}$ & $11 \mathrm{c}$ \\
$\mathrm{IS}$ & $212 \mathrm{a}$ & $64 \mathrm{~b}$ \\
SBS & $176 \mathrm{a}$ & $50 \mathrm{ab}$ \\
STS & $69 \mathrm{c}$ & $36 \mathrm{a}$ \\
\hline
\end{tabular}

${ }^{1} \mathrm{AS}$ - Arcoverde/plantio de sabiá; AL - Arcoverde/plantio de leucena; CL - Caruaru/plantio leucena;

IS - Itambé/plantio de sabiá; SBS - São Bento do Una/plantio de sabiá e STS - Serra Talhada/plantio de sabiá.

*Em cada coluna as médias seguidas pela mesma letra não diferem estatisticamente entre si, pelo teste de Duncan $(\mathrm{p}<0,01)$.

${ }^{\prime} A S$-Arcoverde/sabia plantation; $A L$ - Arcoverdelleucena plantation; $C L$ - Caruaru/leucena plantation;

IS-Itambé/sabia plantation; SBS-São Bento do una/sabia plantation; and STS-Serra Talhada/sabia plantation.

*In each column, means followed by the same letter are not statically different by the Duncan test at $P<0.01$.

A colonização micorrízica variou de $11 \%$ (área CL) a 64\% (área IS). Nas áreas com plantio de leucena, a colonização foi sempre inferior a $15 \%$, enquanto nas áreas com cultivo de sábia houve variação de $36 \%$ a $64 \%$ na colonização (Tabela 2). Estatisticamente, não foi observado diferença na colonização entre as áreas com plantio de leucena (AL e CL), tendo essas diferidas das áreas cultivadas com sabiá. Esse dado poderia 
indicar que há pouca variação na colonização micorrízica em plantas da mesma espécie cultivadas em diferentes locais. Entretanto Balota et al. (1999), estudando os FMAs associados a culturas de mandioca, verificaram que a porcentagem de colonização apresentou valores que variaram de $31 \%$ a $49 \%$ no RJ, $33 \%$ a $46 \%$ em SP e $31 \%$ a $71 \%$ no PR, demonstrando a diferença de colonização em diferentes áreas utilizando-se uma mesma cultura. Fatores relacionados à fertilidade do solo atuam na colonização micorrízica de indivíduos de uma mesma espécie vegetal. Assim, quando observados em diferentes áreas, podem atuar na sua colonização. Abbott e Robson (1991) relataram que o aumento do nível de P no solo pode influenciar espécies de FMAs hábeis a colonizar as raízes das plantas.
Na Tabela 3 foram observadas 24 espécies de FMAs, representando os seguintes gêneros: Acaulospora (6), Archaeospora (1), Entrophospora (1), Gigaspora (3), Glomus (6), Paraglomus (1) e Scutellospora (6). A área CL e STS foram as que apresentaram maior número de espécies (14 espécies), enquanto as áreas AL e AS exibiram menor diversidade (oito espécies).

As espécies Acaulospora scrobiculata, Archaeospora leptoticha e Glomus etunicatum foram encontradas em todas as áreas. Elas parecem estar amplamente distribuídas no Brasil e foram detectadas em vários trabalhos sobre a ocorrência de FMAs em plantas cultivadas (MELO et al., 1997; SOUZA et al., 2002), entre outros.

Tabela 3 - Espécies de fungos micorrízicos arbusculares (FMAs) encontradas nas áreas com plantio de leucena e sabiá no Estado de Pernambuco

Table 3 - FMAs species found in areas with leucena and sabiá plantations in the State of Pernambuco

\begin{tabular}{|c|c|c|c|c|c|c|}
\hline \multirow[t]{2}{*}{ FMA } & \multicolumn{6}{|c|}{ Locais de coleta $^{1}$} \\
\hline & AS & $\mathrm{AL}$ & CL & IS & SBS & STS \\
\hline Acaulospora excavata Ingleby, Walker e Mason & & $\mathrm{X}$ & & & $\mathrm{X}$ & $\mathrm{X}$ \\
\hline A. foveata Trappe e Janos & & $\mathrm{X}$ & & & $\mathrm{X}$ & \\
\hline A. mellea Spain e Schenck & $\mathrm{X}$ & $\mathrm{X}$ & & $\mathrm{X}$ & $\mathrm{X}$ & \\
\hline A. rehmii Sieverding e Toro & & $\mathrm{X}$ & $\mathrm{X}$ & $\mathrm{X}$ & $\mathrm{X}$ & \\
\hline A. scrobiculata Trappe $\quad \mathrm{X}$ & $\mathrm{X}$ & $\mathrm{X}$ & $\mathrm{X}$ & $\mathrm{X}$ & $\mathrm{X}$ & \\
\hline A. tuberculata Janos e Trappe & & & & & $\mathrm{X}$ & \\
\hline Archaeospora leptoticha (Schenck e Smith) Morton e Redecker & $\mathrm{X}$ & $\mathrm{X}$ & $\mathrm{X}$ & $\mathrm{X}$ & $\mathrm{X}$ & $\mathrm{X}$ \\
\hline Entrophospora infrequens (Hall) Ames e Schneider & $\mathrm{X}$ & & & & & \\
\hline Gigaspora decipiens Hall e Abbot & $\mathrm{X}$ & & $\mathrm{X}$ & $\mathrm{X}$ & $\mathrm{X}$ & $\mathrm{X}$ \\
\hline G. gigantea (Nicol. e Gerd.) Gerd. e Trappe & & & $\mathrm{X}$ & & & $\mathrm{X}$ \\
\hline G. margarita Becker e Hall & & $\mathrm{X}$ & & & & \\
\hline Glomus etunicatum Becker e Gerdemann & $\mathrm{X}$ & $\mathrm{X}$ & $\mathrm{X}$ & $\mathrm{X}$ & $\mathrm{X}$ & $\mathrm{X}$ \\
\hline G. glomerulatum Sieverding & & $\mathrm{X}$ & & & & \\
\hline G. macrocarpum Tulasne e Tulasne & $\mathrm{X}$ & $\mathrm{X}$ & $\mathrm{X}$ & & & \\
\hline G. microaggregatum Koske, Gemma e Olexia & & & & $\mathrm{X}$ & & \\
\hline G. microcarpum Tulasne e Tulasne & $\mathrm{X}$ & $\mathrm{X}$ & $\mathrm{X}$ & & & $\mathrm{X}$ \\
\hline G. mosseae (Nicol. e Gerd.) Gerdemann e Trappe & & & & & & $\mathrm{X}$ \\
\hline Paraglomus occultum (Walker) Morton e Redecker & & & $\mathrm{X}$ & & $\mathrm{X}$ & $\mathrm{X}$ \\
\hline Scutellospora erytropa (Koske e Walker) Walker e Sanders & $\mathrm{X}$ & & & & & \\
\hline S. fulgida Koske e Walker & & & $\mathrm{X}$ & $\mathrm{X}$ & & \\
\hline S. gregaria (Schenck e Nicol.) Walker e Sanders & & & $\mathrm{X}$ & $\mathrm{X}$ & $\mathrm{X}$ & $\mathrm{X}$ \\
\hline S. heterogama (Nicol. e Gerd) Gerd. e Trappe & & $\mathrm{X}$ & & & & \\
\hline S. pellucida (Nicol. e Schenck) Walker e Sanders & & & & & & $\mathrm{X}$ \\
\hline S. persica (Koske e Walker) Walker e Sanders & & & & $\mathrm{X}$ & & \\
\hline Total & 8 & 8 & 14 & 9 & 11 & 14 \\
\hline
\end{tabular}


De acordo com Abbott e Robson (1991), existem grandes diferenças entre as espécies de FMAs no que diz respeito aos efeitos das propriedades do solo sobre sua distribuição; algumas espécies ocorrem em solos ácidos ou em solos alcalinos, enquanto outras aparecem tanto em solos ácidos quanto alcalinos. Segundo Stürmer e Bellei (1994), espécies de Acaulospora são mais freqüentemente encontradas em solos ácidos $(\mathrm{pH}<$ 6,2). Gomes e Trufem (1998) encontraram 10 espécies de Acaulospora associadas a solos ácidos ( $\mathrm{pH} 3,2$ e 3,4), na Ilha dos Eucaliptos (São Paulo). Nesse trabalho, o maior número de espécies de Acaulospora (5) foi encontrado em solo com $\mathrm{pH}$ relativamente neutro $(6,4)$ e também em solo ácido (4,9). Silva et al. (2005) relataram a presença de espécies desse gênero em áreas com $\mathrm{pH}$ acima de 7.0. Aparentemente de acordo com o observado neste trabalho (Tabela 1) e em outros, espécies de Acaulospora podem tolerar ampla faixa de $\mathrm{pH}$.

Melo et al. (1997) verificaram a ocorrência de Glomus mosseae em solos onde o $\mathrm{pH}$ apresentou valores médios entre 6,5 e 7,4. Carrenho et al. (2001) também encontraram G. mosseae em áreas com pH médio de 6,9. Aqui essa espécie foi observada apenas na área com maior valor de pH 6,4. De acordo com Schenck e Siqueira (1987), G. mosseae tem preferência por $\mathrm{pH}$ neutro a levemente alcalino. Esse táxon também foi observado apenas em áreas com elevados valores de $\mathrm{pH}(6,2$ a 7,8$)$ por Silva et al. (2005).

Maia e Trufem (1990) observaram a "preferência" de Gigaspora e Entrophospora por solos com pH ácido. Nesse trabalho, Entrophospora infrequens foi encontrada no solo com $\mathrm{pH}$ mais baixo $(4,5)$. Fochi et al. (2004) e Caproni et al. (2005) também verificaram a ocorrência de E. infrequens em solos com pH relativamente ácido.

Com relação à similaridade entre as comunidades, o mais alto índice (79\%) foi encontrado entre as áreas CL e STS, e o mais baixo (35\%), entre as áreas CL e IS (Tabela 4). Embora de acordo com os dados de similaridade de maneira geral não tenha existido predileção de hospedeiros entre as espécies de FMA, foram observadas diferenças qualitativas na comunidade desses fungos quando relacionadas com suas plantas hospedeiras. Acaulospora tuberculata, Entrophospora infrequens, Glomus microagregatum, G. mosseae, Scutellospora erytropa, S. fulgida, S. pellucida e $S$. persica ocorreram apenas nas áreas com plantio de sabiá. Já as espécies Gigaspora margarita, Glomus glomerulatum e Scutellospora heterogama foram verificadas apenas nas áreas com plantio de leucena. Além de características do solo, principalmente $\mathrm{pH}$ e disponibilidade de $\mathrm{P}, \mathrm{Ca}$ e $\mathrm{Mg}$, a compatibilidade entre os simbiontes precisa ser considerada como fator decisivo para a micorrização e a permanência do fungo no agrossistema. Collozi-Filho e Cardoso (2000) observaram diferenças qualitativas na ocorrência de FMAs em relação à planta hospedeira. Gigaspora margarita e G. decipiens ocorreram apenas na crotalária, enquanto Scutellospora heterogama, Acaulospora sp., A. spinosa, A. longula e Glomus diaphanum, apenas no cafeeiro. No entanto, A. scrobiculata, A. appendicula e $S$. gilmorei foram observadas tanto na crotalária quanto no cafeeiro. Os dados observados neste trabalho confirmam que a comunidade de FMA sofre grande influência do hospedeiro e também das condições do solo.

É importante salientar que mais estudos deverão ser realizados para confirmar as tendências observadas neste trabalho, sendo consideradas mais áreas de coletas.

Tabela 4 - Índice de similaridade (índice de Sorensen) de FMA entre as áreas com plantio de leucena e sabiá no Estado de Pernambuco

Table 4 - Index of similarity (index of Sorensen) of FMA between the areas with ofleucena and sabiá plantations in the State of Pernambuco

\begin{tabular}{lcccccc}
\hline Áreas $^{1}$ & AS & AL & CL & IS & SBS & STS \\
\hline AS & 100 & 63 & 55 & 47 & 42 & 46 \\
AL & & 100 & 55 & 35 & 53 & 55 \\
CL & & & 100 & 52 & 64 & 79 \\
IS & & & & 100 & 70 & 52 \\
SBS & & & & & 100 & 72 \\
STS & & & & & & 100 \\
\hline
\end{tabular}

${ }^{1} \mathrm{AS}$ - Arcoverde/plantio de sabiá; AL-Arcoverde/plantio de leucena; CL-Caruaru/plantio leucena; IS - Itambé/plantio sabiá; SBS - São Bento do Una/plantio de sabiá; STS-Serra Talhada/plantio de sabiá. ${ }^{1} \mathrm{AS}$ - Arcoverde/sabia plantation; AL - Arcoverde/leucena plantation; CL-Caruaru/leucena plantation; IS - Itambé/ sabia plantation; SBSSão Bento do una/ sabia plantation; and STS-Serra Talhada/sabia plantation.

\section{CONCLUSÕES}

- Foi observado que espécies de Acaulospora podem tolerar ampla faixa de pH e Entrphospora infrequens tem preferência por solos ácidos, enquanto Glomus mosseae prefere solos neutros à levemente alcalinos.

- Os dados de similaridade confirmam que a comunidade de FMA sofre grande influência do 
hospedeiro e também das condições do solo, já que foram observadas diferenças qualitativas na comunidade desses fungos quando relacionadas com suas plantas hospedeiras e diferentes locais de coletas.

- Os FMAs estão bem distribuídos nas áreas cultivadas com sabiá e leucena, tendo em vista o número de esporos encontrados, o bom percentual de colonização das raízes das plantas e a diversidade observada desses fungos.

\section{AGRADECIMENTOS}

Ao Conselho Nacional de Desenvolvimento Científico e Tecnológico/Fundação de Amparo à Ciência e Tecnologia do Estado de Pernambuco / FACEPE Pernambuco $(\mathrm{CNPq})$ pelo apoio financeiro.

\section{REFERÊNCIAS}

ABBOTT, L. K.; ROBSON, A. D. Factors influencing the occurrence od vesicular-arbuscular mycorrhizas. Agriculture Ecosystems e Environment, v. 35, p.121-150, 1991.

ALMEIDA, R. T.; FREIRE, V. F.; VASCONCELOS, I. Efeitos da interação Glomus macrocarpum, Rhizobium sp. e níveis crescentes de fosfatos de rocha sobre o desenvolvimento de mudas de sabiá (Mimosa caesalpiniaefolia Benth) e de leucena (Leucena leucocephala Lam. de Witt). Ciência Agronômica, v.18, n.1, p.131-136, 1987.

AUGÉ, R. M. et al. Moisture retention properties of a mycorrhizal soil. Plant and Soil, v.230, p.87-97, 2001.

BAKER, A. J. M. et al. The possibility of in situ heavy metal descontamination of polluted soils using crops of metal-accumulating plants. Resources,

Conservation e Recycling, v.11, p.41-49, 1994.

BATINI, M. et al. Resposta da leucena à dupla inoculações rizóbio-fungos micorrízicos e à adubação fosfatadaIn: SIMPÓSIO BRASILEIRO SOBRE MICROBIOLOGIA DOSOLO, 1994, Londrina. Resumos... Londrina: SBMS, 1994. p.93

BALOTA, E. L. et al. Ocorrência de bactérias diazotróficas e fungos micorrízicos arbusculares na cultura de mandioca. Revista Brasileira de Ciência do Solo, v.34, n.7, p.1265-1276, 1999.
CAPRONI, A. L. et al. FMAs em estéril revegetado com Acacia mangium, após mineração de bauxita. Revista Árvore, v.29, n.3, p.373-381, 2005.

CARRENHO, R. et al. Successive cultivation of maize and agricultural practices on root colonization, number of spores and species of arbuscular mycorrhizal fungi. Brazilian Journal of Microbiology, v.32, p.262-270,2001.

COLOZZI-FILHO, A.; CARDOSO, E. J. B. N. Detecção de FMAs em raízes de cafeeiro e de crotalária cultivada na entrelinha. Pesquisa Agropecuária Brasileira, v.35, n.10, p.2033-2042, 2000.

DIAZ, G.; AZCÓN-AGUILAR, C.; HONRUBIA, M. Influence of arbuscular mycorrhizae on heavy metal ( $\mathrm{Zn}$ and $\mathrm{Pb}$ ) uptake and growth of Lygeum spartum and Anthyllis cytisoides. Plant and Soil, v.180, p.331-333, 1996.

FOCCHI, S. S. et al. Fungos micorrízicos arbusculares em cultivos de citros sob manejo convencional e orgânico. Pesquisa Agropecuária Brasileira, v.39, n.5, p.469-476, 2004.

FRANCO, A. A.; FARIA, S. M. The contribution of N2-fixing tree legumes to land reclamation and sustainability in the tropics. Soil

Biology and Biochemistry, v.29, n.5/6, p.897-903, 1997.

GERDEMANN, J. N. Vesicular-arbuscular micorrhiza.. In. TORREY, J.G.; CLARKSON, D.T. (Ed.). The development and function of roots. New York: Academic, 1975. p. 573-579

GERDEMANN, J. W.; NICOLSON, T. H. Spores of mycorrhizal Endogone species extracted from soil by wet sieving and decanting. Transaction of the British Mycological Society v.46, p.235-244, 1963.

GIOVANETTI, M.; MOSSE, B. An evaluation of techniques for measuring vesicular arbuscular mycorrhizal infection in roots. New Phytologist, v.84, p. 489-500, 1980.

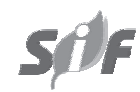

R. Árvore, Viçosa-MG, v.31, n.3, p.427-435, 2007 
GOMES, S. P.; TRUFEM. S. F. B. Fungos micorrízicos arbusculares (Glomales, Zygomycota) na Ilha dos Eucaliptos, Represa do Guarapiranga, São Paulo, SP. Acta Botânica Brasílica, v.12, n.3, p.393-401, 1998.

GUZMAN-PLAZOLA, R. A.; FERRERA-CERRATO, $R$. La endomicorriza vesiculo arbuscular en las leguminosas.

Montecillo: Colegio de Postgraduados, 1990. 122p.

JENKINS, W. R. A rapid centrifugal-flotation technique for separating nematodes from soil. Plant Disease Report, v.48, p.692, 1964.

JESUS, E. C.; SCHIAVO, J. A.; FARIA, S. M. Dependência de micorrizas para a nodulação de leguminosas arbóreas tropicais. Revista Árvore, v.29, n.4, p.545-552, 2005.

KLAUBERG-FILHO, O.; SIQUEIRA, J. O.; MOREIRA, F. M. S. Fungos micorrízicos arbusculares em solos de área poluída com metais pesados. Revista Brasileira de Ciência do Solo, v.26, 125-134, 2002.

MAIA, L. C.; TRUFEM, S. F. B. Fungos micorrízicos vesiculo-arbusculares em solos cultivados no Estado de Pernambuco, Brasil. Revista Brasileira de Botânica, v.13, p.89-95, 1990.

MARSCHNER, H.; DELL, B. Nutrient uptake in mycorrhizal symbiosis. Plant and Soil, v.159, p.89-102, 1994.

MELO, A. M. Y.; MAIA, L. C.; MORGADO, L. B. FMAs em bananeiras cultivadas no vale do submédio São Francisco. Acta Botanica Brasilica, v.11, p.115-121, 1997.

MILLER, R. M.; KLING, M. The importance of integration and scale in the arbuscular mycorrhizal symbiosis. Plant and Soil, v.226, p.295-309, 2000.

MUNYANZIZA, E.; KEHRI, H. K.; BAGYARAJ, D. J. Agricultural intensification, soil biodiversity and agro-ecosystem function in the tropics: the role of mycorrhiza in crops and trees. Applied Soil Ecology, v.6, p.77-85, 1997.

R. Árvore, Viçosa-MG, v.31, n.3, p.427-435, 2007
PHILLIPS, J. M.; HAYMAN, D. S. Improved procedures for clearing roots and staining parasitic and vesicular arbuscular mycorrhizal fungi for rapid assessment of infection.

Transaction of the British Mycological Society, v.55, p.158-161,1970.

SCHENCK, N. C.; PÉREZ, Y. Manual for the identification of VA mycorrhizal fungi, 3.ed. Gainesville: Synergistic-Publications, 1990. 250p.

SCHENCK, N .C.; SIQUEIRA, J. O. Ecology of VA mycorrhizal fungi in temperate agroecosystems. In: SYLVIA, D.M.; HUNG, L.L.; GRAHAM, J.H., (Ed). Mycorrhizae in the Next Decade. NORTH AMERICAN CONFERENCE ON MYCORRHIZAE, 7., 1987,. Proceedings... Gainesville: 1987. p.2-4

SIEVERDING, E. Vesicular-arbuscular mycorrhiza management in tropical agrosystems. Eschborn: Fed. Rep. of Germany: Friedland Bremer, 1991. 371p.

SILVA, G. A. et al. Potencial de infectividade de fungos micorrízicos arbusculares oriundos de área de caatinga nativa e degradada por mineração, no Estado da Bahia, Brasil. Revista Brasileira de Botânica, v.24, p. 135-143, 2001.

SILVA, G. A. et al. Arbuscular mycorrhizal fungi in a semiarid copper mining area in Brazil.

Mycorrhiza, v.15, p.47-53, 2005.

SIQUEIRA, J. O. et al.. Microrganismos e processos biológicos do solo: perspectiva ambiental. Brasilia: Embrapa, 1994. 142p.

SIQUEIRA, J. O. et al. Efeito da formononetina (7 Hidroxi, 4 Metoxi Isoflavona) na colonização micorrízica e crescimento do milho em solo contendo excesso de metais pesados. Revista Brasileira de Ciência do Solo, v.23, p.561-568, 1999.

SIQUEIRA, J. O.; SAGGIN-JUNIOR, O. J. The importance of mycorrhizae association in natural in low fertility. In: MACHADO, A.T.;

MAGNAVACA, R.; PANDEY, S; SILVA, A.F. (Ed.). INTERNATIONAL. SYMPOSIUM ON ENVIRONMENTAL STRESS: MAIZE IN PERSPECTIVE, 1995, Sete Lagoas. Anais... Sete Lagoas: Embrapa, 1995. p. 240-280. 
SORENSEN, T. A method of establishing groups of equal amplitude in plant sociology based on similarity of species content and its application to analysis of the vegetation on Danish commons. In: MCINTOSH, R.P., (Ed.). Phytosociology. Stroudsburg: 1978. p.235-249. (Benchmark Papers in Ecology, 6).

SOUZA, P. V. D. et al. Identificação e quantificação de fungos micorrízicos arbusculares autóctones em municípios produtores de citros no Rio Grande do Sul. Pesquisa Agropecuária Brasileira, v.37, n.4, p.553-558, 2002.
SOUZA, R. G. et al. Diversidade e potencial de infectividade de fungos micorrízicos arbusculares em área de caatinga, na Região de Xingó, Estado de Alagoas, Brasil, Revista Brasileira de Botânica, v.26, n.1, p.49-60, 2003.

STÜMER, S. L.; BELLEI, M. M. Composition and seasonal variation of spore populations of arbuscular mycorrizal fungi in dune soils on the island of Santa Catarina, Brazil.

Canadian Journal of Botany, v.72, p.339-363, 1994. 
